\title{
COMMON FIXED POINTS FOR SEMIGROUPS OF MAPPINGS
}

\author{
ANTHONY T. LAU AND CHI SONG WONG ${ }^{1}$
}

\begin{abstract}
Let $X$ be a compact convex subset of a strictly convex Banach space. Let $S$ be a Hausdorff topological semigroup which is either left amenable or left reversible. Then for any generalised nonexpansive (jointly) continuous action of $S$ on $X, X$ contains a common fixed point of $S$.
\end{abstract}

1. Introduction. Let $S$ be a (nonempty) topological semigroup, i.e. $S$ is a semigroup with a Hausdorff topology such that for each $s$ in $S$, the mappings $x \rightarrow s x$ and $x \rightarrow x s$ of $S$ into $S$ are continuous. Let $C(S)$ be the Banach algebra of all bounded real-valued continuous functions on $S$ with the supremum norm. A function $f$ in $C(S)$ is left uniformly continuous if the mapping $s \rightarrow l_{s} f$, where $l_{s} f(t)=f(s t)$ for all $s, t \in S$, is continuous on $S$ ([12], [13]). Let $\mathrm{LUC}(S)$ be the family of all left uniformly continuous functions on $S$. Then LUC $(S)$ is a Banach subalgebra of $C(S)$ which contains all of the real-valued constant functions on $S$ and is translation invariant [13]. $S$ is left amenable if $\operatorname{LUC}(S)$ has a left invariant mean $\mu$, i.e. $\mu$ is a continuous linear functional on $\operatorname{LUC}(S)$ such that $\|\mu\|=$ $\mu(e)=1$ and $\mu\left(l_{s} f\right)=\mu(f)$ for all $s \in S, f \in \operatorname{LUC}(S)$, where $e$ is the function with $e(s)=1$ for all $s \in S$ [13]. When $S$ is discrete, this definition coincides with that of M. M. Day in [1].

Let $X$ be a subset of a Banach space $B$ with norm $p$. An action of $S$ on $X$ is a mapping of $S \times X$ into $X$, denoted by $(s, x) \rightarrow s x$ such that (st) $x=s(t x)$ for all $s, t \in S, x \in X$ (as a consequence, $S$ can be considered as a family of functions of $X$ into $X$ with the possibility that different elements in $S$ correspond to the same function). A point $x$ in $X$ is a common fixed point of $S$ (with respect to an action) if $s x=x$ for all $s \in S$. An action

Received by the editors March 2, 1973.

AMS (MOS) subject classifications (1970). Primary 47H10; Secondary 54H25.

Key words and phrases. Common fixed points, equicontinuous mapping, generalized nonexpansive mapping, left amenable topological semigroup, left reversible topological semigroup, strictly convex Banach space, strongly almost periodic function.

1 The first author is supported by NRC Grant A7679. The second author is partially supported by NRC Grant A8518 and a grant from the Mathematical Congress. The first author would like to thank Professor T. Rogers for providing a preprint of his joint paper [6] with G. Hardy. 
of $S$ on $X$ is nonexpansive if $p(s x-s y) \leqq p(x-y)$ for all $s \in S, x, y \in X$; it is generalized nonexpansive if there exist nonnegative real numbers $\alpha_{1}, \alpha_{2}, \alpha_{3}, \alpha_{4}, \alpha_{5}$ such that $\alpha_{1}+\alpha_{2}+\alpha_{3}+\alpha_{4}+\alpha_{5}=1$ and for all $s \in S$, $x, y \in X$,

$$
\begin{aligned}
p(s x-s y) \leqq & \alpha_{1} p(x-s x)+\alpha_{2} p(y-s y)+\alpha_{3} p(x-s y) \\
& +\alpha_{4} p(y-s x)+\alpha_{5} p(x-y) .
\end{aligned}
$$

It is obvious that an action of $S$ on $X$ is generalized nonexpansive if it is nonexpansive. The converse is not true even for the case when $X$ is a bounded closed interval [17].

A Banach space $B$ with norm $p$ is strictly convex if for any $x, y, z$ in $B$, $p(x-z)+p(z-y)=p(x-y)$ implies that $z \in[x, y] \quad(=\{(1-t) x+t y$ : $t \in[0,1]\})$. It is the main purpose of this paper to prove the following result. Related results for family of nonexpansive mappings can be found in [3], [7], [11], [15] and [16]. The notion of generalized nonexpansive mappings for metric spaces are considered in [4], [8], [14] and [6].

THEOREM 1. Let $X$ be a compact convex subset of a strictly convex Banach space $B$ with norm $p$. Let $S$ he a left amenable topological semigroup. Then for any (jointly) continuous generalized nonexpansive action of $S$ on $X, S$ has a common fixed point in $X$.

2. Proof of Theorem 1. Since the given action is generalized nonexpansive there exist nonnegative real numbers $\alpha_{1}, \alpha_{2}, \alpha_{-3}, \alpha_{4}, \alpha_{5}$ such that

$$
\alpha_{1}+\alpha_{2}+x_{3}+\alpha_{4}+\alpha_{5}=1
$$

and for all $s \in S, x, y \in X$,

$$
\begin{aligned}
p(s x-s y) \leqq & \alpha_{1} p(x-s x)+\alpha_{2} p(y-s y)+\alpha_{3} p(x-s y) \\
& +\alpha_{4} p(y-s x)+\alpha_{5} p(x-y) .
\end{aligned}
$$

By calculating $(p(s x-s y)+p(s y-s x)) / 2$ through (2), we may without loss of generality assume that $\alpha_{1}=\alpha_{2}$ and $\alpha_{3}=\alpha_{4}$. Thus $\alpha_{1}=x_{2} \leqq \frac{1}{2}, \alpha_{3}=x_{1} \leqq \frac{1}{2}$.

For simplicity, a subset $Y$ of $X$ is said to be invariant if $s y \in Y$ for all $s \in S, y \in Y$. By Zorn's lemma, there exists a minimal nonempty closed convex invariant subset $C$ of $X$. Again by Zorn's lemma, there exists a minimal nonempty invariant closed subset $K$ of $C$. We shall first prove that $s K=K$ for all $s \in S$. Let $x_{0} \in K$. $x_{0}$ will now be used to obtain a measure $\lambda$ on the $\sigma$-algebra $B(K)$ of all Borel subsets of $K$. For each $f$ in $B(K)$, let $T f(s)=f\left(s x_{0}\right), s \in S$. Then $T f \in \operatorname{LUC}(S)[12$, proof of Theorem 1]. Since $S$ is left amenable, $\operatorname{LUC}(S)$ has a left invariant mean $\mu$. Let $\lambda=T^{*} \mu$, where $T^{*}$ is the adjoint of $T$. Then $\|\lambda\|=\lambda(e)=1$ and $\lambda\left({ }_{s} f\right)=\lambda(f)$ for all 
$f \in C(K), s \in S$, where ${ }_{s} f(y)=f(s y), y \in K$. By the Riesz representation theorem, $\lambda$ can be considered as a measure on $B(K)$ with $\lambda(K)=1$ and $\lambda\left(s^{-1}(A)\right)=\lambda(A)$ for all $s \in S, A \in B(K)$, where $s^{-1}(A)=\{y \in K: s y \in A\}$. Since $K$ is compact, the support supp $\lambda$ of $\lambda$ is the smallest compact subset $Y$ of $K$ with $\lambda(Y)=1$. Let $s \in S$. Then from $\lambda\left(s^{-1}(\operatorname{supp} \lambda)\right)=\lambda(\operatorname{supp} \lambda)=1$, we have $s^{-1}(\operatorname{supp} \lambda) \supset \operatorname{supp} \lambda$. So $s(\operatorname{supp} \lambda) \subset s\left(s^{-1}(\operatorname{supp} \lambda)\right) \subset \operatorname{supp} \lambda$ and therefore $\operatorname{supp} \lambda$ is invariant. By minimality of $K$, supp $\lambda=K$. Since $\lambda(s K)=\lambda\left(s^{-1}(s(K))\right) \geqq \lambda(K)=1, s K \supset \operatorname{supp} \lambda=K$. Hence $s K=K$.

Now note that if $K$ is a singleton, then the point in $K$ is a common fixed point of $S$. So we may assume that $K$ contains at least two points.

Case 1. $\alpha_{1}=\alpha_{2}=0$. By Lemma 1 in [3], there exists $z_{0} \in C$ such that

$$
\sup \left\{p\left(z_{0}-x\right): x \in K\right\} \leqq r
$$

for some $r<\delta(K)(=\sup \{p(x-y): x, y \in K\})$. Let

$$
W=\{z \in C: p(z-x) \leqq r \text { for all } x \in K\} .
$$

Then $z_{0} \in W$ and $W$ is a closed convex subset of $C$. To see that $W$ is invariant, let $z \in W, s \in S$. By compactness of $K$, there exists $y_{1} \in K$ such that $p\left(s z-y_{1}\right)=\sup \{p(s z-x): x \in K\}$. Since $s K=K, s y_{2}=y_{1}$ for some $y_{2} \in K$. Now from (2)

$$
\begin{aligned}
p\left(s z-y_{1}\right) & =p\left(s z-s y_{2}\right) \\
& \leqq \alpha_{3} p\left(z-y_{1}\right)+\alpha_{4} p\left(s z-y_{2}\right)+\alpha_{5} p\left(z-y_{2}\right) \\
& \leqq\left(\alpha_{3}+\alpha_{5}\right) r+\alpha_{4} p\left(s z-y_{1}\right) .
\end{aligned}
$$

Since $1-\alpha_{4} \geqq \frac{1}{2}>0$,

$$
p\left(s z-y_{1}\right) \leqq \frac{\alpha_{3}+\alpha_{5}}{1-\alpha_{4}} r=r .
$$

By the choice of $y_{1}, s z \in W$. Thus $W$ is invariant. By minimality of $C$, $W=C$. Hence by definition of $W, \delta(K) \leqq r<\delta(K)$, a contradiction.

Case 2. $\alpha_{1}=\alpha_{2} \neq 0, \alpha_{3}=\alpha_{4} \neq 0$. Let $s \in S$. Then from $s K=K$ and (2),

$$
\begin{aligned}
\delta(K)= & \sup \{p(s x-s y): x, y \in K\} \\
\leqq & \sup \left\{\alpha_{1} p(x-s x)+\alpha_{2} p(y-s y)+\alpha_{3} p(x-s y)\right. \\
& \left.\quad+\alpha_{4} p(y-s x)+\alpha_{5} p(x-y): x, y \in K\right\} \\
\leqq & \alpha_{1} \sup \{p(x-s x): x \in K\}+\alpha_{2} \sup \{p(y-s y): y \in K\} \\
& \quad+\alpha_{3} \sup \{p(x-s y): x, y \in K\}+\alpha_{4} \sup \{p(y-s x): x, y \in K\} \\
& +\alpha_{5} \sup \{p(x-y): x, y \in K\} \\
\leqq & \alpha_{1} \delta(K)+\alpha_{2} \delta(K)+\alpha_{3} \delta(K)+\alpha_{4} \delta(K)+\alpha_{5} \delta(K) \\
= & \delta(K) .
\end{aligned}
$$


Since $\alpha_{1}=\alpha_{2} \neq 0$, we have from (3),

$$
\delta(K)=\sup \{p(x-s x): x \in K\} .
$$

From (4), $s K=K$ and (2),

$$
\begin{aligned}
\delta(K)= & \sup \left\{p\left(s x-s^{2} x\right): x \in K\right\} \\
\leqq & \sup \left\{\alpha_{1} p(x-s x)+\alpha_{2} p\left(s x-s^{2} x\right)\right. \\
& \left.\quad+\alpha_{3} p\left(x-s^{2} x\right)+\alpha_{5} p(x-s x): x \in K\right\} \\
\leqq & \alpha_{1} \delta(K)+\alpha_{2} \delta(K)+\alpha_{3} \delta(K)+\alpha_{5} \delta(K) .
\end{aligned}
$$

Hence $1 \leqq \alpha_{1}+\alpha_{2}+\alpha_{3}+\alpha_{5}<1$, a contradiction.

Case 3. $\alpha_{1}=\alpha_{2} \neq 0, \alpha_{3}=\alpha_{4}=0$. Let $s \in S$. By the Schauder-Tychonoff fixed point theorem, $s w=w$ for some $w \in C$. Since $K$ is compact, there exists $y_{1} \in K$ such that $p\left(y_{1}-w\right)=\sup \{p(x-w): x \in K\}$. Since $s K=K$, $y_{1}=s y_{2}$ for some $y_{2} \in K$. Thus

$$
\begin{aligned}
p\left(w-y_{1}\right) & =p\left(s w-s y_{2}\right) \leqq \alpha_{2} p\left(y_{2}-s y_{2}\right)+\alpha_{5} p\left(w-y_{2}\right) . \\
& \leqq \alpha_{2} p\left(y_{2}-s y_{2}\right)+\alpha_{5} p\left(w-y_{1}\right) .
\end{aligned}
$$

Since $1-\alpha_{5}=\alpha_{1}+\alpha_{2}>0$ and $\alpha_{2} /\left(1-\alpha_{5}\right)=\frac{1}{2}$,

$$
p\left(w-y_{1}\right) \leqq \frac{\alpha_{2}}{1-\alpha_{5}} p\left(y_{2}-s y_{2}\right) \leqq \frac{1}{2} \delta(K) .
$$

By the choice of $y_{1}$,

$$
p(w-x) \leqq \frac{1}{2} \delta(K) \text { for all } x \in K .
$$

By compactness of $K$, there exist $x_{1}, x_{2} \in K$ such that $\delta(K)=p\left(x_{1}-x_{2}\right)$. Now from (5), $\delta(K)=p\left(x_{1}-x_{2}\right) \leqq p\left(x_{1}-w\right)+p\left(w-x_{2}\right) \leqq \delta(K)$, i.e.

$$
p\left(x_{1}-x_{2}\right)=p\left(x_{1}-w\right)+p\left(w-x_{2}\right) .
$$

Since $B$ is strictly convex, we have from (5) and (6), w= $\frac{1}{2}\left(x_{1}+x_{2}\right)$. Since $x_{1}, x_{2}$ do not depend on $w,\left(x_{1}+x_{2}\right) / 2$ is a common fixed point of $S$.

3. Related results. Let $S$ be a topological semigroup. $S$ is left reversible if the family of all closed right ideals in $S$ has the finite intersection property. When $S$ has the discrete topology, $S$ is left reversible if it is left amenable [5, p. 181]. However, in general, "left reversible" and "left amenable" are two independent conditions on a topological semigroup ([1, p. 516], [7, §4]). By using Lemma 1 in [9], we still have $s K=K$ for all $s \in K$ even if, in Theorem $1, S$ is left reversible instead of being left amenable. So we have the following result.

THEOREM 2. Let $X$ be a compact convex subset of a strictly convex Banach space $B$ with norm $p$. Let $S$ be a left reversible topological semigroup. 
Then for any (jointly) continuous generalized nonexpansive action of $S$ on $X$, $S$ has a common fixed point in $X$.

Let $S$ be a topological semigroup. A function $f \in C(S)$ is strongly almost periodic if $\left\{l_{s} f: s \in S\right\}$ is relatively compact in $C(S)$. Let $\mathrm{AP}(S)$ be the family of all strongly almost periodic functions on $S$. Then $\mathrm{AP}(S)$ is a Banach subalgebra of LUC $(S)$ which contains all constant real-valued functions on $S$ and is translation invariant [2]. An action of $S$ on a compact subset $X$ of a Banach space is equicontinuous if $S$ is equicontinuous when it is considered as a family of functions of $X$ into $X$. Now by using Lemma 3.1 in [10] and by modifying the proof of Theorem 1 in an obvious way, we have the following result.

THEOREM 3. Let $X$ be a compact subset of a Banach space $B$ with norm $p$. Let $S$ be a topological semigroup such that $\operatorname{AP}(S)$ has a left invariant mean. Then for any equicontinuous and jointly continuous action of $S$ on $X, S$ has a common fixed point in $X$.

We would like to point out here that by modifying the definitions and proofs in an obvious way, one can prove Theorems $1-3$ for the case when $B$ is a Hausdorff locally convex topological space with its topology induced by a given family of pseudonorms on $B$.

\section{REFERENCES}

1. M. M. Day, Amenable semigroups, Illinois J. Math. 1 (1957), 509-544. MR 19, 1067.

2. K. deLeeuw and I. Glickberg, Application of almost periodic compactification, Acta. Math. 105 (1961), 63-97. MR 24 \#A1632.

3. R. DeMarr, Common fixed points for commuting contraction mappings, Pacific J. Math. 13 (1963), 1139-1141. MR 28 \#2446.

4. M. Edelstein, On fixed and periodic points under contractive mappings, J. London Math. Soc. 37 (1962), 74-79. MR 24 \#A2936.

5. E. E. Granirer, Extremely amenable semigroups, Math. Scand. 17 (1965), 177-197. MR 33 \#5760.

6. G. Hardy and T. Roger, A generalisation of a fixed point theorem of S. Reich, Canad. Math. Bull. (to appear).

7. R. D. Holmes and A. Lau, Nonexpansive actions of topological semigroups and fixed points, J. London Math. Soc. 5 (1972), 330-336.

8. R. Kannan, Some results on fixed points. II, Amer. Math. Monthly 76 (1969), 405-408. MR 41 \#2487.

9. A. T. Lau, Invariant means on subsemigroups of locally compact groups, Rocky Mountain J. Math. 3 (1973), 77-81.

10. - Invariant means on almost periodic functions and fixed point properties, Rocky Mountain J. Math. 3 (1973), 69-76.

11. T. Mitchell, Fixed points of reversible semigroups of nonexpansive maps, Kodai Math. Sem. Rep. 22 (1970), 322-323. MR 42 \#2316. 
12. T. Mitchell, Topological semigroups and fixed points, Illinois J. Math. 14 (1970), 630-641. MR 42 \#5245.

13. I. Namioka, On certain actions of semi-groups on L-spaces, Studia Math. 29 (1967), 63-77. MR 36 \#6910.

14. S. Reich, Some remarks concerning contractive mappings, Canad. Math. Bull. 14 (1971), 121-124. MR 45 \#1145.

15. W. Takahashi, Fixed point theorem for amenable semigroup of nonexpansive mappings, Kodai Math. Sem. Rep. 21 (1969), 383-386. MR 41 \#7501.

16. Chi Song Wong, Fixed point theorems on compact Hausdorff spaces (to appear).

17. - Fixed points of generalised nonexpansive mappings on an inte

Proc. Amer. Math. Soc. (to appear).

Department of Mathematics, University of Alberta, Edmonton, Alberta, CANADA

Department of Mathematics, University of Windsor, Windsor, Ontario, CANADA 